\title{
Computer Modeling of Orthogonal in the Amplified Sense Signal
}

\author{
Aleksandr P. Zhuk \\ Department of Organization and \\ Technology of Information Protection \\ North-Caucasus Federal University \\ Stavropol, Russia \\ alekszhuk@mail.ru
}

\author{
Viktor V. Sazonov \\ Department of Organization and \\ Technology of Information Protection \\ North-Caucasus Federal University \\ Stavropol, Russia \\ vmktor-sazonov@yandex.ru \\ Vladimir P. Pashintsev \\ Department of Information Protectio \\ North-Caucasus Federal University \\ Stavropol, Russia \\ pashintsevp@mail.ru
}

\author{
Dmitrii V. Orel \\ Department of Organization and \\ Technology of Information Protection \\ North-Caucasus Federal University \\ Stavropol, Russia \\ kde.def@gmail.com
}

\begin{abstract}
The article solved the problem of finding analytic dependency showing the influence coefficients of the second diagonal Hermitian matrix by correlation and spectral properties defined by it in the amplified sense of orthogonal signals. The analytical dependences between the coefficients of the second diagonal of the Hermitian matrix and the correlation and spectral characteristics of ensembles of discrete orthogonal signals in the amplified sense are determined. The use of formulas allows for specific selection of ensembles orthogonal in the amplified sense signals, which reduces the time of synthesis. When the values of the diagonal coefficients modules are constant, the arguments of these coefficients do not affect the values of the modules of the unit elements of sets of discrete orthogonal signals in the amplified sense. The value of the eigenvector arguments of a two-diagonal Hermitian matrix is determined by the arguments of its diagonal coefficients. Correlation functions of signals with equal values of modules of unit elements are determined only by the values of their arguments. For certain ratios of modules and arguments of coefficients of second diagonal Hermitian matrix and corresponding minors can be obtained the required levels of lateral peaks of the correlation functions and the values of the relative effective width of the spectrum of the synthesized ensemble of discrete orthogonal in the amplified sense signals.
\end{abstract}

Keywords—Signals sets, orthogonality, correlation, spectrum, Hermitian matrix, two-diagonal matrix.

\section{INTRODUCTION}

In [1], [2] is shown that for any orthogonal basis finite complex space exists Hermitian self-adjoint operator matrix, the eigenvectors which constitute mentioned basis. The coordinates of the eigenvectors of a Hermitian matrix are generally complex numbers, and the orthogonality condition in a complex space coincides with the condition of orthogonality in the amplified sense submitted to analytical discrete complex-conjugate signal $\dot{y}(t)$ and $\dot{z}^{*}(t)$ [1]:

$$
\int_{0}^{T} \dot{y}(t) \cdot \dot{z}^{*}(t) d t=0
$$

where $\dot{y}(t)=a(t) \cdot e^{j \psi_{a}(t)}, \dot{z}^{*}=b(t) \cdot e^{j \psi_{b}^{*}(t)}, a(t)=b(t)$ - the signal amplitude, $\psi_{a}(t)$ and $\psi_{b}^{*}(t)$ - phase of signals, with a second complex conjugate.

\section{Statement of THE PROBlem}

However, the proposed in [1] the method has drawbacks related above all to the lack of analytical relationships between the complex Hermitian matrix coefficients and correlation and spectral characteristics ensembles discrete orthogonal in the amplified sense (EDOSSS) represented by its eigenvectors [3].

The purpose of the article is to determine the analytical relationships between the coefficients of the second diagonal of a Hermitian matrix and the correlation and spectral characteristics of EDOSSS described by its own vectors [4].

\section{EIGENVECTORS HERMITIAN MATRICES}

Consider two-diagonal Hermitian matrix $Q$ self-adjoint operator, presented in exponential form:

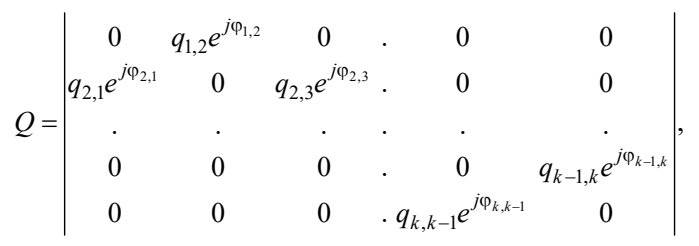

where the values of the coefficients $q_{k-1, k}=q_{k, k-1}$, and arguments $\varphi_{k-1, k}=\varphi_{k, k-1}^{*}$ - complex conjugate

To find the coordinates of the eigenvectors $\left\{\dot{x}_{k}\right\}$. We solve the following algebraic equation:

$$
|Q-\lambda E|=0
$$


where $\lambda$ - eigenvalues; $E$ - identity matrix whose main diagonal in the unit, and the rest zeros.

Expression (3) can be kind of system:

$$
\left\{\begin{array}{l}
-\lambda \dot{x}_{1}+q_{1,2} e^{j \varphi_{1,2}} \dot{x}_{2}=0, \\
q_{2,1} e^{j \varphi_{2,1}} \dot{x}_{1}-\lambda \dot{x}_{2}+q_{2,3} e^{j \varphi_{2,3}} \dot{x}_{3}=0, \\
\ldots \ldots \ldots \ldots, \\
\ldots \ldots \ldots, \\
q_{n, m-1} e^{j \varphi_{n, m-1}} \dot{x}_{m-1}-\lambda \dot{x}_{m}=0 .
\end{array}\right.
$$

Solving the system of equations (4) we obtain the coordinates of the eigenvectors of a Hermitian matrix $Q$ that when $\dot{x}_{1}=\alpha$ will be determined by the expression of the form [2]

$$
\begin{gathered}
\dot{x}_{2}=\alpha \cdot \frac{\lambda}{q_{1,2}} \cdot e^{-j \varphi_{1,2}} ; \\
\dot{x}_{3}=\alpha \cdot \frac{\lambda-q_{1,2}^{2}}{q_{1,2} \cdot q_{2,3}} \cdot e^{-j\left(\varphi_{1,2}+\varphi_{2,3}\right)} ; \\
\dot{x}_{k}=(-1)^{k-1} \cdot \alpha \cdot \frac{\Delta_{k-1}}{q_{12} \cdot q_{23} \cdot \ldots \cdot q_{k-1, k}} \cdot e^{-j\left(\varphi_{1,2}+\varphi_{2,3}+\ldots+\varphi_{k-1, k}\right)}
\end{gathered}
$$

where

$$
\begin{aligned}
& \Delta_{k-1}=\lambda^{k-1}-\lambda^{k-3} \cdot\left(q_{1,2}^{2}+q_{2,3}^{2}+q_{3,4}^{2}+\ldots+\underset{k-1}{q_{k-2}^{2}},\right)+ \\
& +\lambda^{k-5} \cdot\left(q_{1,2}^{2} \cdot q_{3,4}^{2} \cdot \ldots \cdot q_{\substack{k-4, k-3}}^{2} \cdot \underset{k-1}{q_{k-2}}\right)+\ldots+\lambda\left(q_{1,2}^{2} \cdot q_{3,4}^{2} \times \ldots\right. \\
& \left.\ldots \times q_{k / 2+1,}^{2}+\ldots+q_{2,3}^{2} \cdot q_{4,5}^{2} \cdot q_{k-2,}^{2}\right) \\
& \text { - minor, composed of }
\end{aligned}
$$
elements of the first $k-1$ rows and columns of the matrix $\left|Q-\lambda_{k} E\right|, \quad \alpha=a_{n 1} e^{j \psi_{n, 1}}$ - free coordinate, the value of which is chosen arbitrarily.

For a description of coordinate eigenvectors two-diagonal Hermitian matrix in polar coordinates, we introduce variables $a_{k}$ and $\psi_{k}$ represented by expressions of the form:

$$
\begin{aligned}
& a_{k}=(-1)^{k-1} \cdot \alpha \cdot \frac{\Delta_{k-1}}{q_{1,2} \cdot q_{2,3} \cdot \ldots \cdot q_{k-1, k}} \\
& \psi_{k}=\left(\varphi_{1,2}+\varphi_{2,3}+\ldots+\varphi_{k-1, k}\right)
\end{aligned}
$$

From the analysis of expressions (8) that the value for $a_{k}$ influence coefficients modules $q_{k-1, k}$ matrix (2), and $\psi_{k}$ - their arguments $\varphi_{k-1, k}=\varphi_{k, k-1}^{*}$, With all of them depend on the value of the initial phase $\alpha$.

Considering expressions (5-8) system eigenvectors of a Hermitian matrix two-diagonal $Q$ you can submit a matrix $X$ type:

$$
X=\left|\begin{array}{cccc}
a_{11} e^{j \psi_{1,1}} & a_{12} e^{j \psi_{1,2}} & \ldots & a_{1 m} e^{j \psi_{1, m}} \\
a_{21} e^{j \psi_{2,1}} & a_{22} e^{j \psi_{2,2}} & \ldots & a_{2 m} e^{j \psi_{2, m}} \\
\cdot & \cdot & . & \cdot \\
a_{n 1} e^{j \psi_{n, 1}} & a_{n 2} e^{j \psi_{n, 2}} & \ldots & a_{n m} e^{j \psi_{n, m}}
\end{array}\right|
$$

According to [1] the matrix $X$ EDOSSS is a model and each of its eigenvector is one signal of the ensemble, and can be described as a combination of unit cells as follows:

$$
\begin{gathered}
\dot{x}_{y}(t)=\left\{a_{1} e^{j \psi_{1}}, a_{2} e^{j \psi_{2}}, a_{3} e^{j \psi_{3}}, \ldots a_{m / 2-1} e^{j \psi_{m / 2-1}},\right. \\
\left.a_{m / 2} e^{j \psi_{m / 2}}, a_{m / 2+1} e^{j \psi_{m / 2+1}}, \ldots, a_{m} e^{j \psi_{m}}\right\}
\end{gathered}
$$

where $a_{k}$ - the amplitude of a single signal element, $\psi_{k}$ - the phase of a single signal element.

In accordance with [2] with the proviso that the Hermitian matrix coefficients two-diagonal $Q$ $q_{1,2}, q_{3,4}, \ldots q_{k-1, k} \rightarrow \lambda$, but $q_{2,3}, q_{5,6}, \ldots, q_{k-2, k-1} \rightarrow 0$,

Equal amplitudes conditions $a_{1}=a_{2}=a_{3}=\ldots=a_{k}$ can be achieved for all signals in the ensemble. It should be noted that this condition is fulfilled in the presence of different argument values $\varphi_{k-1, k}$ coefficients of the original two-diagonal Hermitian matrix $Q$ form (2), as evidenced by the expression (8).

The case of equality of the amplitudes of signals of the unit cells $a_{1}=a_{2}=a_{3}=\ldots=a_{k}$ It has a value in the practice of the binary signals in digital data transmission systems [5, $6]$.

\section{EFFECT OF COEFFICIENTS OF THE SECOND DIAGONAL \\ HERMITIAN MATRIX BY CORRELATION AND SPECTRAL CHARACTERISTICS EDOSSS}

It is known [7] that the correlation function (CF) signals can be calculated according to the formula of the form:

$$
R_{y z}(\rho)=\frac{1}{m} \sum_{k=\rho+1}^{m} x_{y, k} x_{z, k-\rho}^{*},
$$

where $y$ and $z$ signals in the ensemble represented by their respective unit cells $x_{y}$ and $x_{z}, \rho$ - the number of the side $\mathrm{CF}$ peak [8].

$$
\begin{gathered}
R_{y, z}(\rho)=\frac{1}{m} \cdot \sum_{k=\rho+1}^{m}(-1)^{k-1} \cdot \frac{\alpha \cdot \Delta_{y, k-1}}{q_{1,2} \cdot q_{2,3} \cdot \ldots \cdot q_{k-1, k}} \times \\
\times e^{-j\left(\varphi_{1,2}+\varphi_{2,3}+\ldots+\varphi_{k-1, k}\right)} \cdot(-1)^{k-\rho-1} \times \\
\times \frac{\alpha \cdot \Delta_{z, k-\rho-1}}{q_{1,2} \cdot q_{2,3} \cdot \ldots \cdot q_{k-\rho-1, k-\rho}} \cdot e^{j\left(\varphi_{1,2}+\ldots+\varphi_{k-1-\rho, k-\rho}\right)}= \\
=\frac{1}{m} \sum_{k=\rho+1}^{m}(-1)^{2 k-\rho-2} \frac{\alpha^{2} \cdot \Delta_{y, k-1} \cdot \Delta_{z, k-\rho-1}}{\left(q_{1,2} \cdot \ldots \cdot q_{k-1, k}\right) \cdot\left(q_{1,2} \cdot \ldots \cdot q_{k-\rho-1, \kappa-\rho}\right)} \times \\
\times e^{-j\left(\varphi_{1,2}+\ldots+\varphi_{k-1, k}\right)+j\left(\varphi_{1,2}+\ldots+\varphi_{k-1-\rho, k-\rho}\right)}
\end{gathered}
$$




$$
\begin{gathered}
=\frac{1}{m} \sum_{k=\rho+1}^{m}(-1)^{2 k-\rho-2} \frac{\alpha^{2} \cdot \Delta_{y, k-1} \cdot \Delta_{z, k-\rho-1}}{\left(q_{1,2} \cdot q_{2,3} \cdot \ldots \cdot q_{k-\rho-1, k-\rho}\right)^{2}} \times \\
\quad \times \frac{1}{q_{k-\rho, k-\rho+1} \cdot \ldots \cdot q_{k-1 . k}} \cdot e^{-j\left(\varphi_{k-\rho, k-\rho+1}+\ldots+\varphi_{k-1, k}\right)}
\end{gathered}
$$

where at $y=z$ expression (12) describes the envelope of the autocorrelation function of the signal, and for $y \neq z$ - intercorrelation envelope signal functions.

To assess the spectral characteristics EDOSSS $[9,10]$ use known from [2], the formula of the relative effective width of the spectrum of the signal, provided the energy equation and the reference analysed signals:

$$
\frac{W_{y y}^{2}}{W_{0}^{2}}=\frac{T}{\tau} \cdot\left[1 \pm R_{1}(\tau)\right]
$$

wherein the duration $T$ - signals in the ensemble, $\tau$ - the duration of a single chip, $R_{1}(\tau)$ - the first side peak of the autocorrelation function (ACF) signal.

Based on (12) represent the first side peak in the ensemble ACF signal $[11,12]$ in the following form:

$$
\begin{gathered}
R_{y y}(1)=\frac{1}{m} \cdot \sum_{k=2}^{m}\left[(-1)^{k-1} \cdot \alpha \cdot \frac{\Delta_{y, k-1}}{q_{12} \cdot q_{23} \cdot \ldots \cdot q_{k-1, k}} \times\right. \\
\times e^{-j\left(\varphi_{1,2}+\varphi_{2,3}+\ldots+\varphi_{k-1, k}\right)} \cdot(-1)^{k-2} \cdot \alpha \cdot \frac{\Delta_{y, k-2}}{q_{12} \cdot q_{23} \cdot \ldots \cdot q_{k-2, k-1}} \times \\
\times e^{\left.j\left(\varphi_{1,2}+\varphi_{2,3}+\ldots+\varphi_{k-2, k-1}\right)\right]}=\frac{1}{m} \cdot \sum_{k=2}^{m}\left[(-1)^{2 k-3} \times\right. \\
\left.\times \frac{\alpha^{2} \cdot \Delta_{y, k-1} \cdot \Delta_{y, k-2}}{\left(q_{12} \cdot q_{23} \cdot \ldots \cdot q_{k-2, k-1}\right)^{2} q_{k-1, k}} \cdot e^{-j \varphi_{k-1, k}}\right] .
\end{gathered}
$$

Substituting (14) into (13) obtain an expression

$$
\begin{gathered}
\frac{W_{y y}^{2}}{W_{0}^{2}}=m \pm \sum_{k=2}^{m}\left[(-1)^{2 k-3} \cdot \frac{\alpha^{2} \cdot \Delta_{y, k-1} \cdot \Delta_{y, k-2}}{\left(q_{12} \cdot q_{23} \cdot \ldots \cdot q_{k-2, k-1}\right)^{2} q_{k-1, k}} \times\right. \\
\left.\times e^{-j \varphi_{k-1, k}}\right] .
\end{gathered}
$$

\section{CONCLUSION}

From (7) and (8) implies that for constant values of the diagonal coefficients modules $q_{1,2}, q_{2,3}, \ldots, q_{k-1, k}$, the arguments of these factors do not affect the values of the moduli $a_{k}$ individual elements EDOSSS. Therefore, when any of these arguments, the value of the coefficients $\Delta_{1}, \Delta_{2}, \ldots, \Delta_{k-1, k}$ It will be the same.

Analysis of the relations (7) and (8) demonstrates that the arguments $\psi_{1,2}, \psi_{2,3}, \ldots, \psi_{k-1, k} \quad$ eigenvectors diagonal Hermitian matrix $Q$ defined arguments $\varphi_{1,2}, \varphi_{2,3}, \ldots, \varphi_{k-1, k}$ its diagonal coefficients.CF of signals with equal values of the modules of the unit cells is determined only by the values of their arguments [13-15]. From relations analysis (12, 14, $15)$ it follows that under certain ratios modules $q_{1,2}, q_{2,3}, \ldots, q_{k-1, k}$ and arguments $\varphi_{1,2}, \varphi_{2,3}, \ldots, \varphi_{k-1, k}$ second diagonal coefficients Hermitian matrix and the corresponding minors required levels CF lateral peaks and relative values of the effective width of the spectrum of the synthesized EDOSSS can be obtained.

\section{ACKNOWLEDGMENT}

This work was supported by the Russian Foundation for Basic Research, project No. 18-07-01020.

\section{REFERENCES}

[1] Popenko V. S. "Vector synthesis of orthogonal signal ensembles. Part III". Stavropol, MO RF, 1993 (in Russian).

[2] Ventzel E. S. "Probability theory". Moscow, Academy, 2003 (in Russian).

[3] Orel, D., Zhuk, A., Zhuk, E., Luganskaia, L. "A method of forming code sets for CDMA in communication, navigation and control systems". In: CEUR Workshop Proceedings. 2017.

[4] Pashintsev, V.P., Peskov, M.V., Kalmykov, I.A., Zhuk, A.P., Senokosov, M.A. "Method for the evaluation of ionospheric diffractive and dispersive properties impact on the interference immunity of satellite communication systems". International Journal of Civil Engineering and Technology. 2018; 9(13), pp. 44-61.

[5] Tebueva, F., Kopytov, V., Petrenko, V., Kharechkin, P., Sidorchuk, A. "Method for detecting and eliminating data time series outlier in high-speed process data sensors". International Journal on Communications Antenna and Propagation. 2017; 7(7), pp. 603-612.

[6] Gavrishev, A.A., Zhuk, A.P., Osipov, D.L. "An analysis of technologies to protect a radio channel of fire alarm systems against unauthorized access". In: SPIIRAS Proceedings. 2016.

[7] Varakin L. E. "Theory of signals systems". Moscow, Soviet radio, 1978 (in Russian).

[8] Tebueva, F.B., Kopytov, V.V., Petrenko, V.I., Shulgin, A.O., Demirtchev, N.G. "The identification of data anomalies from information sensors based on the estimation of the correlation dimension of the time series attractor in situational management systems" Journal of Theoretical and Applied Information Technology. 2018; 96(8), pp. 2197-220.

[9] Zhuk, A.P., Ryabtsev, S.S., Khachkizov, R.A., (...), Dzhamiev, N.D., Sherbakov, D.A. "Analysis of the information protection methods in telecommunication systems with channels split by code". In: CEUR Workshop Proceedings. 2254, pp. 303-310.

[10] Dytso, A., Egan, M., Perlaza, S.M., Vincent Poor, H., Shamai, S "Optimal inputs for some classes of degraded wiretap channels". 2018 IEEE Information Theory Workshop, ITW 2018 \#8613368.

[11] Kopytov, V.V., Petrenko, V.I., Tebueva, F.B., Streblianskaia, N.V. "An improved Brown's method applying fractal dimension to forecast the load in a computing cluster for short time series". Indian Journal of Science and Technology. 2016; 9(19): Article \#93909.

[12] Wang, B., Mu, P., Li, Z. "Artificial-noise-aided beamforming design in the MISOME wiretap channel under the secrecy outage probability constraint". IEEE Transactions on Wireless Communications. 2017; 16(11), 8016587, pp. 7207-7220

[13] Khouzani, M.H.R., Malacaria, P. "Relative perfect secrecy: Universally optimal strategies and channel design". Proceedings IEEE Computer Security Foundations Symposium. 2016; August \#7536367.

[14] Ganshyn, D.G., Dudka, A.A., Bitchenko, A.N., Tsopa, A.I. "Analysis of structural secrecy of multi-frequency signals of broadband communication systems". Telecommunications and Radio Engineering (English translation of Elektrosvyaz and Radiotekhnika). 2016; 75(13), pp. 1209-1219.

[15] Pashintsev, V.P., Kalmykov, I.A., Zhuk, A.P., Kalmykov, M.I., Rezenkov, D.N. "Application of spoof resistant authentication protocol of spacecraft in low earth orbit systems of satellite communication". International Journal of Mechanical Engineering and Technology. 9(5), pp. 958-965. 